\title{
Mechanisms of Protein Import into Mitochondria
}

\author{
Rosemary A. Stuart, Hideyu Ono ${ }^{\S}$, Thomas Langer, and Walter Neupert* \\ Adolf Butenandt Institut für Physiologische Chemie der Universität München, Goethestraße 33, 80336 Mün- \\ chen, Germany
}

Our understanding of mitochondrial biogenesis has been considerably broadened over the past five years or so, following the identification of numerous components involved in the targeting and translocation of proteins to and across the mitochondrial membrane system. The vast majority of mitochondrial proteins are encoded in the nucleus and are synthesized as precursor proteins containing the necessary sequence information to target them specifically to the mitochondrion. Import of these proteins across the mitochondrial membrane(s) can occur in a post-translational manner. Mitochondria contain two distinct membranes, an outer and inner membrane, so preproteins targeted to the mitochondrial matrix must traverse both of these membranes. Two independent protein translocation machineries, one in each of these membranes (TOM, translocase of the outer membrane and TIM, translocase of the inner membrane) facilitate these translocation events. Translocation of preproteins across the inner membrane and their subsequent folding to native conformation in the matrix are both facilitated by molecular chaperones.

\section{Mitochondrial targeting signals}

Most nuclear encoded proteins bear N-terminal extensions, mitochondrial targeting sequences (presequences) which contain all necessary information to target the proteins specifically to the mitochondria. Proteins destined for the outer membrane and some of those of the intermembrane space and inner membrane are exceptions, as they bear no additional targeting sequences.

The N-terminal presequences are exclusively borne by precursors which must either insert into or traverse the inner membrane. In most cases these sequences are cleavable by the mitochondrial processing peptidase (MPP) following import into the matrix. These mitochondrial-trageting sequences are rich in positivelycharged amino acid residues. Their potential secondary structure, namely an amphipathic, positively charged $\alpha$ helix, appears to be a common crucial feature for target-

\footnotetext{
* To whom correspondence should be addressed.

Fax: +49-89-5996-270, Tel.: +59-89-5996-312/313

e-mail: Neupert@bio.med.uni-muenchen.de

$\&$ Present address: Department of Biochemistry, Yamagata University, School of Medicine, Yamagata 990-23, Japan
}

ing and import into mitochondria (35). The presequences initiate translocation across the inner membrane in a membrane potential $(\Delta \Psi)$ dependent fashion (30). Preproteins bearing these $\mathrm{N}$-terminal sequences become translocated across the inner membrane in a linear fashion, i.e. in an $\mathrm{N}$ - to C-terminal direction.

On the other hand not all proteins which are imported in a $\Delta \Psi$-dependent fashion contain $\mathrm{N}$-terminal cleavable matrix-targeting sequences, e.g. some proteins of the inner membrane. The import mechanism(s) of such preproteins are largely unknown, as it is very difficult to define internal mitochondrial targeting signals. Recently though an in depth study of the import mechanism of one such protein, BCS1 protein has provided some insight about the nature and operation of internal targeting signals (10). The BCS1 protein, an inner membrane protein, is synthesized in the cytosol without an $\mathrm{N}$-terminal targeting signal, in fact the $\mathrm{N}$-terminus has a net negative charge (23). Instead, a stretch of positively charged amino acid residues located immediately after the single transmembrane domain has the potential to form a positively-charge amphipathic helix and serves to target the BCS1 to the mitochondria. The neighbouring transmembrane domain is essential for import, and it seems to interact with the apolar face of the amphipathic helix to form a hairpin loop structure. The internal targeting signal penetrates the TIM machinery in the form of this loop structure rather than in a linear fashion as described for the N-terminal targeted proteins (10).

Such a targeting and translocation mechanism mediated by internal targeting signals which form loops, may indeed operate for many other proteins synthesized without $\mathrm{N}$-terminal presequences, including those which contain a number of transmembrane domains.

\section{The role of cytosolic factors in mitochondrial protein import \\ In order to become translocated across the mitochon- drial outer and inner membranes, a preprotein must adopt an unfolded conformation. Some preproteins in the cytosol may fold to a native-like structure which must become unfolded upon membrane translocation, whilst others may be prevented from premature fold- ing. The presence of an $\mathrm{N}$-terminal cleavable prese-}


quence may partially interfere with such a folding process in the cytosol. On the other hand, various cytosolic factors have been identified that stabilize non-native precursors against aggregation prior to import, and some in addition contribute to efficient mitochondrial targeting.

Cytosolic Hsp 70 proteins and the DnaJ homologue, Ydjlp. The first proteins proposed to ensure preproteins remain in a translocation competent state were the cytosolic Hsp70 (ct-Hsp70) proteins, the SSa proteins $(6,9)$. Genetic depletion of the levels of the Ssalp ctHsp70 protein resulted in the cytosolic accumulation of a mitochondrial precursor protein. It was thereby concluded that these molecular chaperones played an important role in efficient mitochondrial import of precursors. Recently a direct interaction between a preprotein destined for the mitochondrial outer membrane, Tom70, and ct-Hsp70 has been demonstrated (31). Further support for the role of ct-Hsp70s in mitochondrial protein import came from the analysis of a temperature-sensitive (ts) yeast mutant of the DnaJ homologue present in the cytosol, Ydj1p (3). At restrictive temperature conditions the cytosolic accumulation of a number of mitochondrial preproteins was reported. Ydj1p triggers the release of Ssa1p-bound substrate proteins in vitro $(7,8)$. It has been postulated to perform a similar function in vivo and thereby facilitating the release of proteins from bound Ssa1p at the mitochondrial surface.

Mitochondrial import stimulating factor (MSF). MSF, a heterodimeric complex purified from rat liver cytosol and rabbit reticulocyte lysate was shown to stimulate protein import into mitochondria $(11,24)$. A member of the 14-3-3 protein family, MSF is thought to specifically recognize mitochondrial preproteins and to target them to the mitochondrial receptor proteins. The Tom70-Tom37-receptor complex in the mitochondrial outer membrane has been identified as the binding site for the MSF-associated preprotein. Release of the bound preprotein from MSF requires ATP hydrolysis and is an NEM-sensitive process (15).

Consequently MSF has a dual function, firstly as a novel chaperone maintaining the precursor in an translocation competent state and secondly in conferring specificity on the mitochondrial targeting process. Recently two homologues of the mammalian MSF have been described in yeast, the BMH1 and $\mathrm{BMH} 2$ proteins (34). Evidence for the role of these proteins in assisting mitochondrial protein import in yeast has not yet been presented.

\section{Translocation of preproteins across the outer mem- brane}

The recognition of preproteins at the mitochondrial surface and their translocation across the outer mem- brane is mediated by a complex of proteins known as the TOM complex (for recent review see 17). A number of the components of this complex have been identified and cloned, mainly from the fungi, Neurospora crassa and Saccharomyces cerevisiae. A functional characterization of these proteins lead to the identification of the receptors involved in the initial recognition of preproteins on the mitochondrial surface. The Tom70-Tom71Tom37 receptor system is used by a few specific preproteins, whilst the vast majority (including all the presequence bearing proteins) are directly recognized by the Tom20-Tom 22 receptor system. Those proteins which are bound first by Tom70-Tom71-Tom 37 system, then become transferred to the Tom20-Tom22 system. All proteins pass through a common translocation pore which is thought to be comprised of the remaining subunits of the TOM complex.

The TOM complex directly facilitates the insertion of proteins into the outer membrane and the passage of a few proteins into the intermembrane space. The translocation process of presequence-targeted proteins is initiated only by the TOM complex. Binding of the N-terminal parts of the preprotein to intermembrane space-exposed elements of the TOM complex is thought to stabilize an unfolded state of the precursor on the mitochondrial surface $(17,22)$. The presequence now exposed to the intermembrane space, interacts directly with components of the inner membrane translocation machinery, the TIM complex, in a $\Delta \Psi$-dependent manner. By acting to maintain an unfolded state of the preprotein, the outer membrane import apparatus apparently supports the action of the matrix-localized mitochondrial Hsp70 system in unfolding preproteins at the mitochondrial surface.

\section{Translocation preproteins across the inner membrane is driven by matrix-localized chaperones}

Translocation of proteins across the inner membrane requires a membrane potential and an ATP-dependent proteinaceous translocation machinery at its inner surface $(25,29)$. This machinery involves the co-ordinated action of the matrix-located Hsp70 molecular chaperone (mt-Hsp70) (14). Tim44, a peripheral component of the translocation pore of the inner membrane (19) and Mge1p, a yeast homologue of the bacterial GrpE (16, 37). All of these proteins perform an essential function in mitochondrial biogenesis and hence their presence is required for viability of the cell.

Driven by the ATP-dependent action of mt-Hsp70, protein translocation across the inner membrane occurs via what is thought to be a passive pore constituted of other components of TIM complex, Tim 17 and Tim 23 and possibly two further proteins.

The presequence of preproteins spontaneously inserts across the TIM pore in response to $\Delta \Psi$ and only upon 
binding to the mt-Hsp70-Tim44 dependent import machinery does it become stabilized there. By binding in such a manner, retrograde movement of preproteins out of the translocation pore is prevented, thereby conferring unidirectionality upon the import process. The association of mt-Hsp70/Tim44 complex with the translocating polypeptide chain is regulated by ATP and the Mge1p.

Our present understanding of this nucleotide-modulated cycle of events is summarized as follows. MtHsp70 bearing a bound ATP associates with Tim44 at the matrix opening of the TIM pore. Binding of the emerging translocating preprotein stimulates ATP hydrolysis by $\mathrm{mt}-\mathrm{Hsp} 70$, which in turn results in its dissociation from Tim44. Mt-Hsp70 now in the ADP-bound conformation, a substrate high-affinity state, remains bound to the preprotein. Released from Tim44, the translocating polypeptide is now free to slide in the TIM pore, however is prevented from undergoing retrograde movement due to the presence of the bound mt-Hsp70. Further binding of Tim44 and another ATP-loaded mtHsp70 to more C-terminal segments of the polypeptide serve to ensure another cycle of its translocation. Recycling of the ADP-loaded mt-Hsp70 from the polypeptide is prompted by Mgelp (37). By acting as an nucleotide exchange factor, Mge1p replaces the bound ADP for an ATP, thereby converting mt-Hsp70 back to a lower affinity state, resulting in the release from its bound substrate. This fresh ATP-loaded form of mt-Hsp70 is available for rebinding to $\operatorname{Tim} 44(32,37)$. At later stages of the import process, mt-Hsp70 can bind directly to the matrix-exposed segments of the polypeptide independently of Tim44. Recently experimental evidence for these two distinct preprotein-bound populations of mt-Hsp70, Tim44 associated and non-associated, has been presented (33).

\section{Folding and assembly of proteins following import into the mitochondrial matrix}

Following translocation across the mitochondrial membrane system as an extended polypeptide chain, once in the matrix imported proteins must fold correctly to attain their native functional conformation. In many cases this folding process is an assisted one, involving the cooperation of mt-Hsp70 and sometimes transfer to another matrix-localized chaperone, Hsp60.

The chaperone function of mt-Hsp70 during folding is influenced by Mdj1p (the mitochondrial DnaJ homologue; 28) and Mge1p. The folding of newly imported proteins is impaired following their import into mitochondria lacking the Mdj1p protein. Mdj1p can act directly on the mt-Hsp70 system, as it is required for efficient binding of unfolded polypeptides to $\mathrm{mt}-\mathrm{Hsp} 70$ $(26,36)$. In addition, Mdj1p prevents aggregation of mitochondrial proteins in a manner independent form
mt-Hsp70 action. Defective folding of newly imported proteins occurs in mitochondria isolated from a temperature sensitive mutant form of Mge1p (37).

The mitochondrial Hsp60 system. Mt-Hsp60, the mitochondrial homologue of E. coli GroEL plays an important role in protein folding in the mitochondrial matrix. Mt-Hsp60 was originally identified in a genetic screen for yeast mutants displaying a defect in the enzymatic activity of ornithine transcarbamoylase, a protein imported into mitochondria. Folding and assembly of both imported and mitochondrially encoded proteins is impaired upon deletion of functional mt-Hsp60 $(4,5$, 12). Under thermal stress conditions, mt-Hsp60 maintains mitochondrial proteins in their enzymatic conformation by preventing aggregation of thermolabile proteins (21).

Newly imported proteins interact with mt-Hsp70 and mt-Hsp60 in a sequential manner (20). Mt-Hsp60 acts in an ATP-dependent manner and is regulated by mtHsp10, a homologue of $\operatorname{GroES}(13,18,27)$. In a temperature-sensitive yeast mutant of mt-Hsp10, folding of newly imported proteins was found to be impaired under restrictive conditions (13). Not all imported proteins require the mt-Hsp60 system for folding though. The particular requirement of the specific chaperone systems for assisting protein folding may depend on the preprotein and its specific properties.

\section{REFERENCES}

1. Berthold, J., Bauer, M.F., Schneider, H.C., Klaus, C., Dietmeier, K., Neupert, W., and Brunner, M. 1995. Cell, 81: $1085-1093$.

2. Blom, J., Dekker, P.J.T., and MeiJer, M. 1995. Eur. J. Biochem., 332: 309-314.

3. Caplan, A., Cyr, D.M., and Douglas, M.G. 1992. Cell, 71: 1143-1155.

4. Cheng, M.Y., Hartl, F.-U., Martin, J., Pollock, R.A., Kalousek, F., Neupert, W., Hallberg, E.M., Hallberg, R.L., and HoRWICH, A.L. 1989. Nature, 337: 620-625.

5. Cheng, M.Y., Harte, F.-U., and Horwich, A.L. 1990. Nature, 348: 455-458.

6. Chirico, W., Waters, M., and Blobel, G. 1988. Nature, 332: 805-810.

7. Cyr, D.M., Lu, X., and Douglas, M.G. 1992. J. Biol. Chem., 267: 20927-20931.

8. CYR, D.M. 1995. FEBS Lett., 359: 129-132.

9. Deshaies, R.J., Koch, B.D., Werner-Washburne, M., Craig, E.A., and SCHEKMAN, R. 1988. Nature, 332: 800-805.

10. Fölsch, H., Guiard, B., Neupert, W., and Stuart, R.A. 1996. EMBO J., 15: 479-487.

11. HachiYa, N., KomiYa, T., Alam, R., IWahashi, J., Sakaguchi, M., Omura, T., and Mihara, K. 1994. EMBO $J .$, 13: 5146-5154.

12. Hallberg, E.M., Shu, Y., and Hallberg, R.L. 1993. Mol. Cell Biol., 13: 3050-3057.

13. Höhfeld, J. and Harte, F.-U. 1994. J. Cell Biol., 126: 305315 . 
14. Kang, P.-J., Ostermann, J., Shilling, J., Neupert, W., Craig, E.A., and Pfanner, N. 1990. Nature, 348: 137-143.

15. Komiya, T., SaKaguchi, M., and Mihara, K. 1996. EMBO J., 15: 399-407.

16. Layoraya, S., Dekker, P.J.T., Voos, W., Craig, E.A., and Pfanner, N. 1995. Mol. Cell Biol., 15: 7098-7105.

17. Lill, R. and NeUpert, W. 1996. Trends Cell Biol., 6: 56-61.

18. Lubben, T.H., Gatenby, A.A., Donaldson, G.K., Lorimer, G.H., and Vitanen, P.V. 1990. Proc. Natl. Acad. Sci. USA, 87: 7683-7687.

19. MaArse, A.C., Blom, J., Grivell, L.A., and Meijer, M. 1992. EMBO J., 11: 3619-3628.

20. Manning-Krieg, U.C., Scherer, P.E., and Schatz, G. 1991. EMBO J., 10: 3273-3280.

21. Martin, J., Horwich, A.L., and Harte, F.-U. 1992. Science, 258: 995-998.

22. Mayer, A., Neupert, W., and Lill, R. 1995. Cell, 80: 127137.

23. Nobrega, F.G., Nobrega, M.P., and Tzagoloff, A. 1992. EMBO J., 11: 3821-3829.

24. Ono, H. and Tuboi, S. 1988. J. Biol. Chem., 263: 3188-3193.

25. Pfanner, N., Craig, E.A., and MeiJer, M. 1994. Trends Biochem. Sci., 19: 368-372.

26. Prip-Buus, C., Westermann, B., Schmitt, M., Langer, T., NeUPert, W., and SchWARZ, E. 1996. FEBS Lett., 380: 142146.
27. Rospert, S., Glick, B.S., Jenö, P., Schatz, G., Todd, M.T., Lorimer, G.H., and Vittanen, P.V. 1993. Proc. Natl. Acad. Sci. USA, 90: 10967-10971.

28. Rowley, N., Prip-Buus, C., Westermann, B., Brown, C., Schwarz, E., Barrell, B., and NeUpert, W. 1994. Cell, 77: 249-259.

29. Ryan, K.R. and JENSEN, R.E. 1995. Cell, 83: 517-519.

30. SChleYer, M., Schmidt, B., and NeUPeRt, W. 1982. Eur. J. Biochem., 125: 109-116.

31. Schlossmann, J. and Neupert, W. 1995. J. Biol. Chem., 270: 27116-27121.

32. SChNeIder, H.C., Berthold, J., BaUer, M.F., Dietmeier, K., Guiard, B., BrunNer, M., and Neupert, W. 1994. Nature (Lond.), 371: 768-774.

33. Ungermann, C., Neupert, W., and Cyr, D.M. 1996. EMBO J., 15: 735-744.

34. Van Heusden, G.P.H., Griffiths, D.J.F., Ford, J.C., China-Woeng, T.F.C., Schrader, P.A.T., Carr, A.M., and SteEnSMA, H.Y. 1995. Eur. J. Biochem., 229: 45-53.

35. VON HeIJNe, G. 1986. EMBO J., 5: 1335-1342.

36. Wagner, I., ArLt, H., Van Dyck, L., LaNGer, T., and NeUPERT, W. 1994. EMBO J., 13: 5135-5145.

37. Westermann, B., Prip-Buus, C., Neupert, W., and SCHWARZ, E. 1995. EMBO J., 14: 3452-3460. 